\title{
Ergodico in letteratura
}

\author{
Lucia Francalanci
}

PUBBLICATO: 23 APRILE 2021

$\mathrm{T}$ ra le varie forme ed espressioni appartenenti al fenomeno della letteratura elettronica (o letteratura digitale), una delle più interessanti, nonostante sia ancora poco conosciuta, è la letteratura ergodica.

In ambito letterario, il termine ergodico è stato proposto per la prima volta dallo studioso norvegese Espen Aarseth nel suo libro Cybertext. Perspectives on Ergodic Literature (1997), il quale riprende l'aggettivo dal lessico specialistico della fisica (si veda più avanti). Nel suo volume, Aarseth critica i tradizionali concetti di narrativa e interattività, lanciandosi invece in una campagna a favore del cybertesto e del testo ergodico. La definizione di letteratura ergodica proposta dallo studioso non è particolarmente chiara e, più in generale, dalla sua analisi non risulta evidente quale sia l'effettiva differenza tra cybertestualità ed ergodicità: "nella letteratura ergodica è richiesto uno sforzo non banale per consentire al lettore di attraversare il testo. Se la letteratura ergodica deve avere un senso come concetto, deve esserci anche della letteratura non-ergodica, nella quale lo sforzo di attraversare il testo è superficiale, senza responsabilità extranoematiche a carico del lettore eccetto (per esempio) il movimento degli occhi e il periodico o arbitrario voltare le pagine" (trad. e corsivi miei).

Cercando di chiarire le sue parole, potremmo definire la letteratura ergodica come una letteratura che presenta una struttura narrativa e un'impaginazione non propriamente lineari, e che comporta un'interazione attiva con il lettore, il quale deve compiere uno sforzo supplementare per poter fruire dell'opera. Tale sforzo è chiamato extranoematico. Il termine noematico deriva da noema (dal gr. nóema 'pensiero'), uno dei tecnicismi usati in linguistica per indicare l'unità minima di significato (sul modello di morfema e fonema); lo sforzo extranoematico è dunque quello che non riguarda la decodifica/interpretazione del significato espresso verbalmente, ma che si va ad aggiungere ad essa.

Qualsiasi testo richiede un certo tipo di interazione e di partecipazione al lettore, sia per quanto riguarda l'approccio più prettamente "fisico" alla lettura sia per quanto riguarda la funzione interpretativa. Il testo ergodico richiede al lettore un ulteriore impegno, che può andare da una richiesta di maggiore attenzione (ad esempio per l'impaginazione: il testo può essere infatti disposto da destra verso sinistra, dal basso verso l'altro, in diagonale, invertito, riflesso, annidato a vari livelli, inserito in una forma circolare o in altre figure, ecc.), fino alla ricostruzione stessa della storia (talvolta la vicenda si dipana su più livelli di narrazione che si intersecano tra loro - una storia nella storia nella storia - e il lettore diventa parte attiva nella ricomposizione degli avvenimenti; in alcuni volumi è addirittura il lettore stesso a scegliere quali capitoli leggere o quale percorso seguire). 

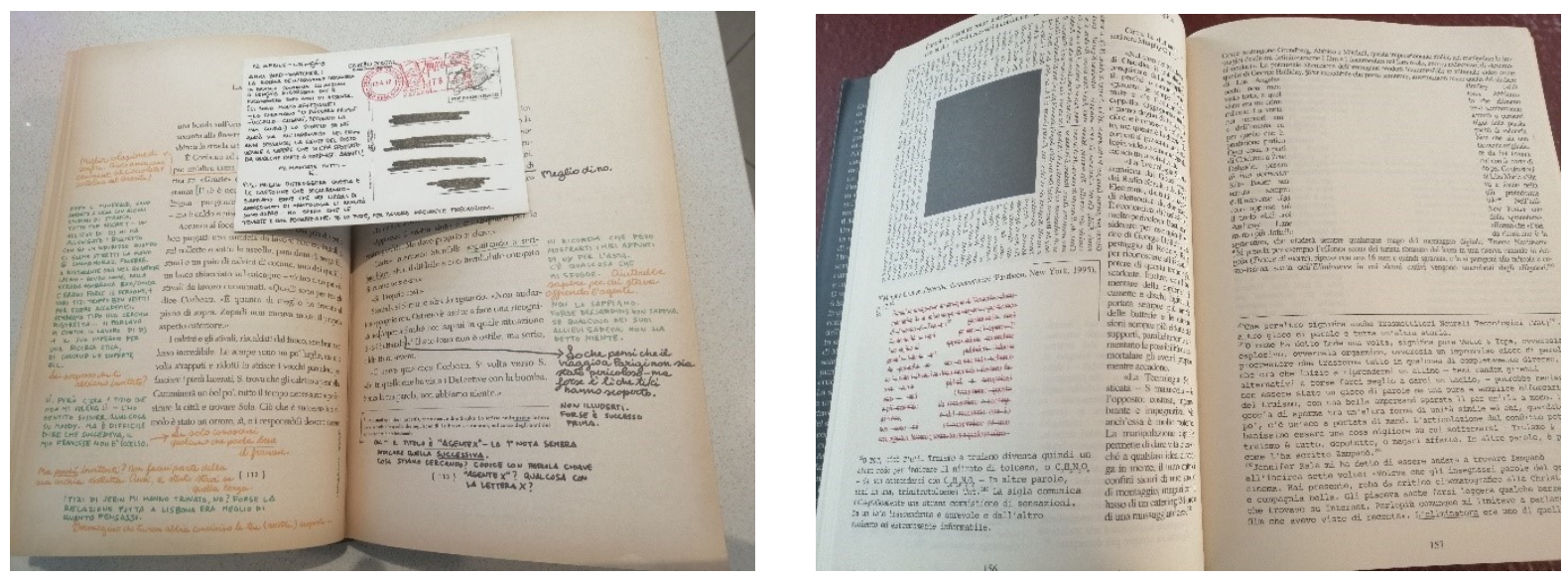

Si tratta di una letteratura sperimentale, dai confini non ben marcati; l'ergodicità può assumere svariate forme ed è per questo che gli esempi che si possono rintracciare sono molto diversi tra loro. Anzi, proprio tale diversificazione non rende ben chiaro quali siano i tratti che determinano l'appartenenza o meno alla categoria di testo ergodico. Tra gli esempi proposti da Aarseth, i primi risalgono addirittura all'antichità, come il testo sacro cinese I Ching, conosciuto anche come Il libro dei mutamenti, e risalente all'epoca della dinastia Chou occidentale (ıг22-770 a.C.). Il libro, utilizzato a scopo divinatorio, è composto da 64 simboli, o esagrammi, le cui combinazioni forniscono le risposte a domande di tipo matematico, filosofico o fisico. Altri esempi indicati dall'autore sono i Calligrammi (I9I3-19i6) di Guillaume Apollinaire, un genere di poesie in cui le parole formano diverse figure, senza però seguire una sequenza chiara che determini l'ordine di lettura, e Centomila miliardi di poesie di Raymond Queneau (I96I), un libretto di soli io fogli (tagliati in I4 strisce orizzontali, ognuna contenente un verso) che permette di comporre innumerevoli sonetti a piacimento (il libro viene considerato anche tra gli esempi di letteratura combinatoria).

Altri casi di letteratura ergodica sono considerati il volume Composizione n. 1 di Marc Saporta (Composition n. 1, 1962; ed. italiana 1962), in cui le pagine non sono numerate e rilegate (il lettore può mescolarle come carte da gioco e disporle come preferisce, ricavandone ogni volta una storia diversa), oppure, tra i più recenti, Casa di foglie di Mark Z. Danielewski (House of leave, 200o; ed. italiana 2005), S. La nave di Teseo di Doug Dorst e J.J. Abrams (S. Ship of Theseus, 2013; ed. italiana 2014), IlMistero.doc di Matthew McIntosh (theMystery.doc, 20I7; ed. italiana 20I7), ecc.

Nonostante si possano rintracciare esempi di testi ergodici già in epoca antica, è soltanto in anni più recenti che il termine ergodico ha fatto la sua comparsa in ambito letterario. Come abbiamo visto, Aarseth lo usa per la prima volta nel suo libro Cybertext del 1997 , prendendolo in prestito dalla fisica. La parola era stata infatti introdotta dal matematico e fisico austriaco Ludwig Boltzmann (I844-I906), per riferirsi a un "sistema o processo i cui valori medi calcolati nel tempo coincidono statisticamente con i valori medi calcolati su un grande numero di sistemi o processi uguali” (GRADIT).

I dizionari inglesi (Collins Dictionary, Oxford Dictionary, Merriam-Webster Dictionary) registrano ergodic 'ergodico' (e il derivato ergodicity 'ergodicità) esclusivamente nell'accezione che ha in ambito matematico-fisico, datandolo 1926. Anche i dizionari italiani marcano l'aggettivo come tecnicismo della fisica e riconducono la sua origine alla voce greca ergódès, propriamente 'difficile' (Devoto-Oli online, Garzanti, Supplemento 2004 del GDLI, GRADIT, Hoepli e Sabatini-Coletti). Nel suo articolo sull'etimologia di ergodico (Ergodico: storia di un'etimologia, "Lingua nostra", LIX, I998, pp. 89-90), F.M. Pontani fa invece risalire l'aggettivo al tedesco ergodish, a sua volta derivato del sostantivo femminile die Ergode, che indica un particolare tipo di sistema meccanico. Secondo lo studioso, i due termini (ergodish e Ergode) sono stati coniati da Boltzmann nel i 885 (Ueber die Eigenschaften monocyklischer und 
anderer damit verwandter Systeme, in "Journal für die reine und angewandte Mathematik", XCVIII, I884-I885, pp. 68-94), il quale però nel suo articolo non ne dichiara l'etimologia; tuttavia, il genere femminile potrebbe indicare che Ergode deriva dalla composizione delle parole greche épyov 'energia'

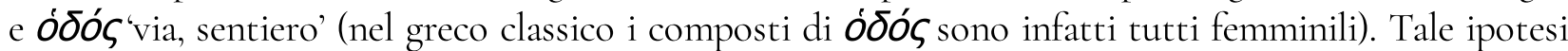
è ripresa dallo Zingarelli, che registra ergodico (dal 2004) come "voce dotta, dal tedesco ergodish, agg. di Ergode, n. coniato da L. Boltzmann con i due elementi gr. érgon 'opera' e hodós 'via', intendendo "passaggio di energia"; anche il Vocabolario Treccani online riporta tale etimologia greca, senza però far riferimento alla voce tedesca. I dizionari etimologici non registrano la voce ergodico ma soltanto la base ergo-, primo elemento di composti propri della fisica e della tecnologia con il significato di 'opera, lavoro' (ergologia, ergometria, ergonomia, ergotécnica, ergoterapia).

I dizionari dell'uso non sono concordi neppure sulla data di prima attestazione di ergodico: si va dal I920 (Zingarelli), al 1956 (Devoto-Oli, GRADIT), al I987 (Sabatini-Coletti).

Il sostantivo ergodicitá, nel senso di 'proprietà di un sistema o processo ergodico' è invece accolto, sempre come termine specialistico della fisica, soltanto dal Devoto-Oli online (datato XX secolo), dal GRADIT (I987), dal Vocabolario Treccani online e dallo Zingarelli (senza data).

L'uso di ergodico e di ergodicitá in ambito letterario non viene dunque registrato, né dai dizionari inglesi, né da quelli italiani. La presenza dell'accezione fisico-matematica non rende facile effettuare ricerche dirimenti circa la prima attestazione in italiano dell'aggettivo nell'uso che ci interessa, né tanto meno rintracciare in rete i dati relativi alla sua effettiva diffusione. Per limitare il rumore nelle interrogazioni dei motori di ricerca (Google) e degli archivi dei quotidiani, si è scelto quindi di affiancare a ergodico alcune delle forme che co-occorrono maggiormente, come, "letteratura", "testo", "libro" (le ricerche legate al sostantivo ergodicità generano invece troppo rumore).

Tale espediente ci permette di provare a ricostruire la storia dell'accezione letteraria della parola nella nostra lingua: la prima occorrenza del termine in ambito letterario che siamo riusciti a individuare risale al 200I, in un articolo che si occupa di poesia intertestuale, pubblicato sulla rivista elettronica di letteratura italiana "Bollettino '9oo" dell'Università di Bologna (lo stesso articolo compare anche nella rivista "il Verri", n. I6, 200I, pp. 50-64):

Per caratterizzare questa letteratura, vorrei riprendere la definizione di letteratura ergodica (ergon: lavoro, in greco), che Espen Aarseth trae dal campo della matematica, segnalando che questa letteratura non si limita agli ipertesti stricto sensu, ma comprende anche i generatori di testi e la poesia animata, dal momento che questi fanno ricorso, in un modo o in un altro, all'attività di un interattore. [...] Dal punto di vista dell'autore, la scelta di un dispositivo ergodico comporta, se non addirittura la scomparsa dell'autore in quanto tale, la perdita volontaria di una parte dei suoi poteri di scrittore, e l'abbandono di una concezione classica della letteratura, considerata come espressione di singoli sentimenti o di un pensiero costituito. [...] Al di là della tentazione del multiplo, la scelta di un dispositivo ergodico risponde anche all'antico desiderio di coinvolgere il lettore nell'elaborazione dell'opera e di aprire un dialogo con lui. (Jean Clément, Elementi di poetica ipertestuale, in "Bollettino '9oo", n. I, 200I)

Nei testi a stampa si trovano attestazioni dell'aggettivo anche negli anni successivi, soprattutto in volumi dedicati alla scrittura o alla critica letteraria e quasi sempre in riferimento all'espressione coniata da Aarseth:

A questi sistemi si riferisce Espen Aarseth, altro teorico della cyberspace textuality, introducendo la definizione di letteratura o arte "ergodica", ovvero quel tipo di opere elettroniche "prodotte da qualche tipo di sistema cibernetico, per esempio una macchina (o una persona), che opera come meccanismo di risposta continuo [information feedback loop] che genererà una diversa sequenza semiotica ogniqualvolta è 
azionato". (Domenico Fiormonte, Scrittura e filologia nell'era digitale, Torino, Bollati Boringhieri, 2003, pp. I45-I46)

La prima occorrenza in rete si ha nel 2008, in un articolo pubblicato sul blog Libri Senza Carta dedicato alla manifestazione letteraria BookCamp, svoltasi a Rimini nel luglio di quell'anno:

Il primo gruppo, in ordine di tempo, al quale abbiamo partecipato, si è occupato invece di nuove scritture sul web, affrontando un tema tanto affascinante quanto ancora poco conosciuto come quello della letteratura ergodica (dal greco ergon "opera" e odós "via", "percorso"), termine coniato da Espen J. Aarseth nel suo libro Cybertext: Perspectives on Ergodic Literature (1997). Questo tipo di letteratura, a differenza di quella tradizionale che si basa su una lettura diacronica (dal greco "attraverso il tempo": dia e chrónos), cioè storica o sequenziale, che segue un ordine di tempo, richiede un intervento attivo del lettore, che si sceglie un suo percorso personalizzato, giungendo ad esiti imprevedibili. (Valeria Bellagamba, La letteratura del futuro a Rimini, librisenzacarta.it, 20/7/2008)

Si tratta però di un caso isolato e fino al 20Ir le attestazioni in rete sono quasi inesistenti. Dal 20II l'aggettivo inizia a diffondersi, con un aumento più significativo a partire dal 20I7. Come ci mostra Google Trends, un'impennata nelle ricerche da parte degli utenti web si ha a fine 20r9; questo dato potrebbe essere collegato alla ristampa del volume Casa di foglie di Mark Z. Danielewski, pubblicato per la prima volta in Italia da Mondadori nel 2005 (l'edizione statunitense è del 2000), poi divenuto introvabile (e molto ricercato) e ripubblicato da 66thandznd proprio nel novembre del 2019.

- Letteratura ergodica Argomento
: $\quad+$ Confronta

Italia $\quad$ Ultimi 5 anni $\checkmark \quad$ Tutte le categorie $\checkmark \quad$ Ricerca Google

Interesse nel tempo

$\pm\langle<$

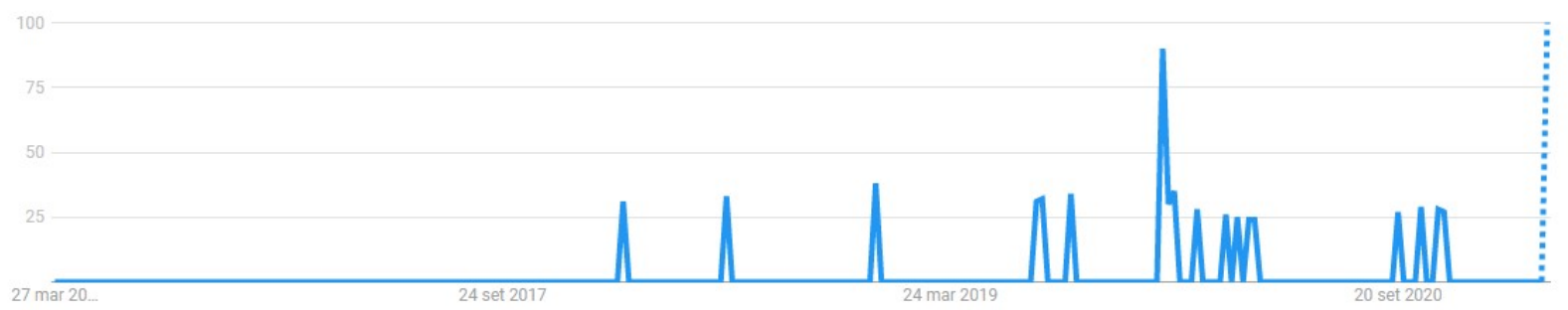

Anche sui quotidiani le rarissime attestazioni dell'aggettivo, sempre nell'accezione letteraria, si hanno a partire dal 20r9: due occorrenze di ergodica nell'archivio del "Corriere della Sera" (una del 2org e una del 2020) e una sola occorrenza di ergodico in quello di "Repubblica" (del 2020):

Un simile cammino è spettato a Casa di foglie, l'esordio di Mark Z. Danielewski. Apparso nel 2000 negli Usa, battezzato da Stephen King «il Moby-Dick dell'horror», è un romanzo maestoso di 760 pagine, emblema della letteratura ergodica contemporanea, corrente in cui ogni elemento (stile, forma, immagini), si esprime allo stremo affinché si provochi nel lettore affaticato l'elevazione, il disorientamento, la ricerca degli indizi e infine il dubbio sull'eventuale veridicità delle notizie contenute. (Orazio Labbate, Il tatuatore e la casa infestata di buchi neri, "la Repubblica", 3/II/2019) 
Se si tentasse di incasellare certe opere sperimentali dentro una famiglia di riferimento, si rischierebbe di perdere la loro complessità che, invece, meriterebbe soltanto di essere contemplata attraverso una lettura matta e disperatissima. Parecchi i degni romanzi di questo genere. William Burroughs con Nova Express, in cui la trama disturbante non segue la logica del realismo bensì la schizofrenia di un incubo; Casa di foglie di Mark Z. Danielewski, un volume che rientra tra i più sensazionali esempi della letteratura ergodica (cioè una letteratura nella quale il lettore è invitato a prendere parte attiva oltre la semplice lettura del testo); Ultime storie e altre storie ed Europe Central di William T. Vollmann che, benché costruisca fitte trame storiche, imbastisce, contrariamente alla struttura granitica, un efficace stile pregno di esoterismo e di contenuti fantafilosofici [...]. (Orazio Labbate, Il mistero del mistero del romanzo sognato, "la Repubblica", 2/2/2020)

È strano immergersi in questi giorni nelle quasi milleseicento pagine de IlMistero.doc di Matthew McIntosh, mirabilmente tradotte da Luca Fusari. Si comincia con un tizio di nome Daniel che si risveglia in un letto sconosciuto, accanto a una donna sconosciuta, e si rende conto di aver perso la memoria. Pare che sia uno scrittore e che stia lavorando da undici anni a un romanzo intitolato IlMistero.doc. [...] Il tutto nel perfetto pastiche ergodico che risale ai tempi di Sterne e fu nobilitato negli anni Novanta dal post-moderno: la sfida al lettore che è chiamato a un impegno che trascende il puro godimento del testo. (Giancarlo De Cataldo, La storia di tutte le storie, "la Repubblica", sez. Robinson, I $/ 5 / 2020$, p. II)

In generale, si nota che la presenza della parola in rete è comunque piuttosto contenuta, prova del fatto che il fenomeno della letteratura ergodica risulta ancora poco conosciuto. Le pagine in italiano di Google (in data 25/3/202I) restituiscono infatti 3.200 risultati per "letteratura ergodica" (nessuna occorrenza per la forma plurale), 94 risultati per "testo ergodico" (4I per il plurale), 323 per "libro ergodico" (8I per il plurale).

In una precedente indagine, effettuata a gennaio 202I, era stato possibile rintracciare anche rari esempi dell'uso sostantivato dell'aggettivo, per indicare gli autori - gli ergodici - dei volumi appartenenti a questo filone narrativo; ad oggi, la medesima ricerca non restituisce alcun risultato.

\section{Cita come:}

Lucia Francalanci, Ergodico in letteratura, "Italiano digitale", XVII, 2021/2 (aprile-giugno)

DOI: $10.35948 / 2532-9006 / 2021.7520$

Copyright 2021 Accademia della Crusca

Pubblicato con licenza creative commons CC BY-NC-ND 